Commun. Korean Math. Soc. 28 (2013), No. 3, pp. 581-587

http://dx.doi.org/10.4134/CKMS.2013.28.3.581

\title{
GENERIC DIFFEOMORPHISMS WITH ROBUSTLY TRANSITIVE SETS
}

\author{
Manseob Lee and Seunghee Lee
}

ABSTRACT. Let $\Lambda$ be a robustly transitive set of a diffeomorphism $f$ on a closed $C^{\infty}$ manifold. In this paper, we characterize hyperbolicity of $\Lambda$ in $C^{1}$-generic sense.

\section{Introduction}

A fundamental problem in differentiable dynamical systems is to understand how a robust dynamic property (that is, a property that holds for a system and all $C^{1}$ nearby ones) on the underlying manifold would influences the behavior of the tangent map on the tangent bundle. In this paper, we study the robust dynamic property for a transitive set. Let $M$ be a closed $C^{\infty}$ manifold, and let $\operatorname{Diff}(M)$ be the space of diffeomorphisms of $M$ endowed with the $C^{1}$-topology. Denote by $d$ the distance on $M$ induced from a Riemannian metric $\|\cdot\|$ on the tangent bundle $T M$. Let $f \in \operatorname{Diff}(M)$ and $\Lambda$ be a closed $f$-invariant set. The set $\Lambda$ is transitive if there is a point $x \in \Lambda$ such that $\omega(x)=\Lambda$. Here $\omega(x)$ is the forward limit set of $x$. Denote by $\left.f\right|_{\Lambda}$ the restriction of $f$ to the set $\Lambda$. A maximal invariant set of $f$ in an open set $U$, denoted by $\Lambda_{f}(U)$, is the set of points whose whole orbit contained in $U$, that is,

$$
\Lambda_{f}(U)=\bigcap_{n \in \mathbb{Z}} f^{n}(U)
$$

A set $\Lambda$ is locally maximal in $U$ if there is a compact neighborhood $U$ of $\Lambda$ such that $\Lambda=\Lambda_{f}(U)$. Note that if $\Lambda$ is locally maximal in $U$, then for any compact neighborhood $V$ of $\Lambda, \Lambda_{f}(V)=\Lambda_{f}(U)=\Lambda$. Now, we introduce the notion of the robustly transitive set.

Definition 1.1. We say the set $\Lambda$ is robustly transitive set (or, $\left.f\right|_{\Lambda}$ is robustly transitive) if

(i) there is a neighborhood $U$ of $\Lambda$ and a $C^{1}$-neighborhood $\mathcal{U}(f)$ of $f$ such that $\Lambda_{f}(U)=\Lambda=\bigcap_{n \in \mathbb{Z}} f^{n}(U)$ (that is, $\Lambda$ is locally maximal),

Received June 27, 2012; Revised March 28, 2013.

2010 Mathematics Subject Classification. 34D30, 37C20.

Key words and phrases. transitive set, robustly transitive set, generic, dominated splitting, partially hyperbolic, hyperbolic. 
(ii) for any $g \in \mathcal{U}(f), \Lambda_{g}(U)=\bigcap_{n \in \mathbb{Z}} g^{n}(U)$ is transitive for $g$.

Note that a circle rotation $f_{\theta}$ is transitive if $\theta$ is irrational, but $f_{\theta}$ is not robustly transitive. To see this notice that if $\theta$ is rational, then every point in $\mathbb{S}^{1}$ is periodic under $f_{\theta}$.

A diffeomorphism $f \in \operatorname{Diff}(M)$ is transitive if there is a point $x \in M$ such that $\omega(x)=M$. Analogously, $f$ is robustly transitive if every $g C^{1}$-closed to $f$ also is transitive. By the definition of the robustly transitive set, we know that robust transitivity is an open condition. It is an important research area of the global dynamics. In fact, there is a great deal of complexity seen in these systems that is still not well understood. In the case of two dimensional systems many things are fairly well understood, but in case of higher dimensional systems there are many things which are unknown.

From now, we introduce nonhyperbolic type robustly transitive diffeomorphisms. First, Mañé [8] constructed that there exists a diffeomorphism $f$ on the three dimensional torus $\mathbb{T}^{3}$ satisfies for the diffeomorphism $f \in \operatorname{Diff}\left(\mathbb{T}^{3}\right)$, there is a $C^{1}$-neighborhood $\mathcal{U}(f)$ of $f$ such that every $g \in \mathcal{U}(f)$ is transitive, but not Anosov. Second, Shub [11] constructed the nonhyperbolic robustly transitive diffeomorphisms in the four dimensional torus $\mathbb{T}^{4}=\mathbb{T}^{2} \times \mathbb{T}^{2}$ by perturbing the product of a derive from Anosov(DA) diffeomorphisms and an Anosov, where derive from Anosov diffeomorphisms is defined on the two torus $\mathbb{T}^{2}$ which are obtained via saddle-node bifurcations of Anosov systems - the unfolding of the bifurcation derives to the derive form Anosov diffeomorphisms whose nonwandering set is a source and a nontrivial hyperbolic attractor (see [12]). Note that the derive from Anosov diffeomorphisms on $\mathbb{T}^{2}$ construct robustly nonhyperbolic transitive (see [4]). Finally, Bonatti and Díaz [2] shown that one can perturb the product of any diffeomorphism $F$ having a hyperbolic transitive attractor $\Lambda_{F}$ and the identity $i d$ on any closed manifold to obtain $H C^{1}$-closed to $F \times i d$ with a robustly nonhyperbolic transitive attractor $\Lambda_{H}$. Especially, $\Lambda_{H}=\Lambda_{H}(U)$ for some neighborhood $U$, and $\Lambda_{H}(U)$ is robustly transitive. In particular, all robustly transitive diffeomorphisms are nonisotopic to the identity, but there are robustly nonhyperbolic transitive diffeomorphisms isotopic to the identity. Actually, in [2], the author constructed that given any transitive Anosov vector field $X$, let $X_{t}$ be the flow of $X$ at $t \in \mathbb{R}$. Then we can perturb $X_{t}$ to obtain a robustly transitive diffeomorphisms.

From the above fact, one can obtain robustly nonhyperbolic transitive sets via cycles containing periodic points of different indices (dimension of the stable manifold). Therefore, we see that the nonhyperbolic robustly transitive set is always contain periodic points with different indices and coincide with the closure of their transverse homoclinic points (i.e., the transverse intersections between the invariant manifolds of a periodic point) (see [5]). From the facts, in this paper, we study that if the any dimensional robustly transitive diffeomorphisms satisfies some condition then it is hyperbolic. 
We say that $\Lambda$ is hyperbolic if the tangent bundle $T_{\Lambda} M$ has a $D f$-invariant splitting $E^{s} \oplus E^{u}$ and there exist constants $C>0$ and $0<\lambda<1$ such that

$$
\left\|\left.D_{x} f^{n}\right|_{E_{x}^{s}}\right\| \leq C \lambda^{n} \text { and }\left\|\left.D_{x} f^{-n}\right|_{E_{x}^{u}}\right\| \leq C \lambda^{-n}
$$

for all $x \in \Lambda$ and $n \geq 0$.

Recall that an invariant set $\Lambda$ is basic set if it is hyperbolic, locally maximal and the set of periodic points in $\Lambda$ is dense in $\Lambda$. The following theorem by Mañé ([9]) motivates our main theorem.

Theorem 1.2. Let $M$ be a surface and $\Lambda$ be a robustly transitive set. Then $\Lambda$ is a basic set.

Note that in one dimension, there is not robustly transitive diffeomorphism. Because, the diffeomorphisms with finitely many hyperbolic periodic points are open and dense in $\operatorname{Diff}\left(\mathbb{S}^{1}\right)$.

We say that $\Lambda$ is dominated splitting if the tangent bundle $T_{\Lambda} M$ has a $D f$ invariant splitting $E \oplus F$ and there exist constants $m>0$ and $0<\lambda<1$ such that

$$
\left\|\left.D_{x} f^{m}\right|_{E_{x}}\right\| \cdot\left\|\left.D_{x} f^{-m}\right|_{F_{f}{ }_{(x)}}\right\| \leq \lambda
$$

for all $x \in \Lambda$. A $D f$-invariant bundle $E$ defined on $\Lambda$ is called uniformly contracting (resp. expanding) if there are constants $C>0$ and $\lambda \in(0,1)$ such that for every $n>0$, one has

$$
\left.\left\|D_{x} f^{n}(v)\right\| \leq C \lambda^{n}\|v\| \quad \text { (resp. } \quad\left\|D_{x} f^{-1}(v)\right\| \leq C \lambda^{n}\|v\|\right)
$$

for all $x \in \Lambda$ and $v \in E$. The set $\Lambda$ is called partially hyperbolic if there is a dominated splitting $T_{\Lambda} M=E \oplus F$ such that either $E$ is uniformly contracting or $F$ is uniformly expanding. $([6])$.

Next, in case of 3-dimension manifolds, Díaz et al. proved the following

Theorem 1.3. Let $M$ be a three dimensional manifold. If a diffeomorphism $f \in \operatorname{Diff}(M)$ is robustly transitive, then $f$ is partially hyperbolic.

Finally, in [3], Bonatti et al. dealt with the case of arbitrary dimension.

Theorem 1.4. On any dimensional manifold $M$, if a diffeomorphism $f \in$ $\operatorname{Diff}(M)$ is robustly transitive, then $f$ admits a dominated splitting.

The stable manifold $W^{s}(p)$ and the unstable manifold $W^{u}(p)$ are defined as following. It is well known that if $p$ is a hyperbolic periodic point of $f$ with period $k$, then the sets

$$
\begin{array}{r}
W^{s}(p)=\left\{x \in M: f^{k n}(x) \rightarrow p \quad \text { as } n \rightarrow \infty\right\} \text { and } \\
W^{u}(p)=\left\{x \in M: f^{-k n}(x) \rightarrow p \text { as } n \rightarrow \infty\right\}
\end{array}
$$

are $C^{1}$-injectively immersed submanifolds of $M$.

It is clear that robustly transitive set enjoys some property of hyperbolicity such as uniform hyperbolicity, partial hyperbolicity or dominated splitting. In 
this paper, on arbitrary dimension manifold, in generic context (see definition below), we will study the relationship among robust transitivity, hyperbolicity and the following condition.

Condition $(P)$ : Let $\Lambda$ be a closed invariant set. For any hyperbolic periodic points $p, q$ in $\Lambda$, we have

$$
W^{s}(p) \cap W^{u}(q) \neq \emptyset, \quad \text { and } \quad W^{u}(p) \cap W^{s}(q) \neq \emptyset .
$$

Here, $W^{s}(p)$ and $W^{u}(p)$ are the stable manifold of $p$ and the unstable manifold of $p$, respectively.

A subset $\mathcal{G} \subset \operatorname{Diff}(M)$ is called residual if it contains a countable intersection of open and dense subsets of $\operatorname{Diff}(M)$. A property is called $C^{1}$ generic if it holds in a residual subset of $\operatorname{Diff}(M)$. We use the terminology for $C^{1}$-generic to express there is a residual subset $\mathcal{G} \subset \operatorname{Diff}(M)$, and if $f \in \mathcal{G}$. The following is main theorem in this paper.

Theorem 1.5. Let $\Lambda$ be a robustly transitive set. For $C^{1}$ generic $f$, if $f$ satisfies condition $(P)$, then $\Lambda$ is hyperbolic.

\section{Proof of Theorem 1.5}

Let $M$ be arbitrary dimensional manifold, and $f \in \operatorname{Diff}(M)$. The following theorem by Mañé [9] will be crucial to prove our main theorem.

Theorem 2.1. Let $\Lambda$ be a closed $f \in \operatorname{Diff}(M)$-invariant, and let $\left.f\right|_{\Lambda}$ be robustly transitive. The following statements are equivalent:

(i) there is a $C^{1}$-neighborhood $\mathcal{U}(f)$ of $f$ such that for any $g \in \mathcal{U}(f)$, any periodic point of $\Lambda_{g}(U)=\bigcap_{n \in \mathbb{Z}} g^{n}(U)$ is hyperbolic and has the same index;

(ii) there is a $C^{1}$-neighborhood $\mathcal{U}(f)$ of $f$ such that for any $g \in \mathcal{U}(f), \Lambda_{g}(U)$ is hyperbolic.

Note that if $p$ is a hyperbolic periodic point of $f$, then there is a $C^{1}$ neighborhood $\mathcal{U}(f)$ and a neighborhood $U$ of $p$ such that for all $g \in \mathcal{U}(f)$, there is a unique hyperbolic periodic point $p_{g} \in U$ of $g$ with the same period as $p$ and $\operatorname{index}\left(p_{g}\right)=\operatorname{index}(p)$. Here index $(p)=\operatorname{dim} E_{p}^{s}$, and the point $p_{g}$ is called the continuation of $p$.

Lemma 2.2. There exists a residual set $\mathcal{G}_{1} \subset \operatorname{Diff}(M)$ such that for any $f \in$ $\mathcal{G}_{1}$, we have the following property. For any $C^{1}$-neighborhood $\mathcal{U}(f)$ of $f$ there is $g \in \mathcal{U}(f)$ such that $g$ has two distinct hyperbolic periodic points $p_{g}, q_{g} \in P(g)$ with different indices then $f$ has two distinct hyperbolic periodic points $p, q \in$ $P(f)$ with different indices.

Proof. Take a countable basis $\mathcal{B}=\left\{U_{n}\right\}_{n \in \mathbb{N}}$ of $M$. For each $U_{n} \in \mathcal{B}$, we defined by $\mathcal{H}_{n}$ the set of all diffeomorphisms $f$ such that $f$ has a $C^{1}$-neighborhood $\mathcal{U}(f)$ of $f$ with the following properties: for any $g \in \mathcal{U}(f)$, there are $p_{g}, q_{g} \in U_{n}$, in 
distinct periodic hyperbolic points of $g$ with different indices. Then it is clear that $\mathcal{H}_{n}$ is open in $\operatorname{Diff}(M)$ for each $n \in \mathbb{N}$. Let

$$
\mathcal{N}_{n}=\operatorname{Diff}(M)-\overline{\mathcal{H}_{n}} .
$$

Then $\mathcal{H}_{n} \cup \mathcal{N}_{n}$ is an open and dense subset of $\operatorname{Diff}(M)$. Put

$$
\mathcal{R}_{n}=\bigcap_{n \in \mathbb{N}} \mathcal{H}_{n} \cup \mathcal{N}_{n},
$$

is a residual subset of $\operatorname{Diff}(M)$. Then

$$
\mathcal{G}_{1}=\bigcap_{n \in \mathbb{N}} \mathcal{R}_{n},
$$

is also a residual subset of $\operatorname{Diff}(M)$. Let $f \in \mathcal{G}_{1}$. Suppose that for any $C^{1}$ neighborhood $\mathcal{U}(f)$ of $f$, there exist $g \in \mathcal{U}(f)$ and $U_{n} \in \mathcal{B}$ such that $p_{g}$ and $q_{g}$ in distinct hyperbolic periodic points of $g$ with different indices which are elements in $U_{n}$. Then $f \in \overline{\mathcal{H}_{n}}$. Since $f \in \mathcal{G}_{1}$ we know that $f \in \mathcal{H}_{n}$. Thus there are $p, q$ in distinct hyperbolic periodic points of $f$ with different indices. Moreover, if $\Lambda$ is a closed $f$-invariant locally maximal in $U$, then there is $k \in \mathbb{N}$ such that $\Lambda \subset U_{k} \subset U$. For $\mathcal{U}(f)$, there is $g \in \mathcal{U}(f)$ and two hyperbolic periodic points $p_{g}$ and $q_{g} \in P(g)$ with different indices such that $p_{g}, q_{g} \in U_{k} \subset U$. From the above proof, $f$ having two distinct hyperbolic periodic points $p$ and $q$ with different indices. Since $\Lambda$ is locally maximal in $U, p, q \in \Lambda$.

Now, we introduce the notion of the weak eigenvalue (see [13]). Let $p$ be a periodic point $f$. For $0<\delta<1$, we say $p$ has a $\delta$-weak eigenvalue if $D_{p} f^{\pi(p)}$ has an eigenvalue $\lambda$ such that $(1-\delta)^{\pi(p)}<|\lambda|<(1+\delta)^{\pi(p)}$. Note that if $p$ is a hyperbolic periodic point of $f$, then $p$ does not have the $\delta$-weak eigenvalue. Because if $p$ has a $\delta$-weak eigenvalue, then by Franks' Lemma, there exist a diffeomorpshism $g C^{1}$-nearby $f$ such that the periodic point $p_{g}$ has an eigenvalue, say $\lambda$, and $|\lambda|=1$. Thus this is a contradiction by the stability theorem.

Remark 2.3 ([1, Lemma 5.1(2)]). There exists a residual set $\mathcal{G}_{2} \subset \operatorname{Diff}(M)$ such that if $f \in \mathcal{G}_{2}$ then fix $\delta>0$, if for every neighborhood $\mathcal{U}(f)$ of $f$ there exist $g \in \mathcal{U}(f)$ and $p_{g} \in P_{h}(g)$ with a $\delta$-weak eigenvalue then there exists $p \in P_{h}(f)$ with a $2 \delta$-weak eigenvalue, where $P_{h}(f)$ is the set of hyperbolic periodic points of $f$.

Lemma 2.4 ([10, Lemma 2.4]). Let $\Lambda$ be locally maximal in $U$, and let $\mathcal{U}(f)$ be a $C^{1}$-neighborhood of $f$. If for any $g \in \mathcal{U}(f), p \in \Lambda_{g}(U) \cap P(g)$ is not hyperbolic, then there is $g_{1} \in \mathcal{U}(f)$ possessing hyperbolic periodic points $q_{1}$ and $q_{2}$ in $\Lambda_{g_{1}}(U)$ with different indices, where $\Lambda_{g_{1}}(U)=\bigcap_{n \in \mathbb{Z}} g_{1}^{n}(U)$.

Proposition 2.5. There exists a residual set $\mathcal{G}_{3} \subset \operatorname{Diff}(M)$ such that if $f \in \mathcal{G}_{3}$, and $\Lambda$ is the robustly transitive set for $f$ which satisfies condition $(P)$, then there exist $\delta>0$ such that no point in $\Lambda$ has a $\delta$-weak eigenvalue. 
Proof. Let $\mathcal{G}_{3}$ be the residual given by the intersection $\mathcal{G}_{1}$ and the set of KupkaSamle diffeomorphisms. We will derive a contradiction. Let $f \in \mathcal{G}_{3}$, and $\mathcal{U}(f)$ be a $C^{1}$-neighborhood of $f$. Suppose that for $n \in \mathbb{N}$, there is $p_{n} \in \Lambda \cap P(f)$ such that $p_{n}$ has a $1 / n$-weak eigenvalue. By Franks' Lemma and Lemma 2.4, there exists $g \in \mathcal{U}(f)$ such that $g$ have two distinct periodic orbits $p_{g}$ and $q_{g} \in$ $\Lambda_{g}(U)=\bigcap_{n \in \mathbb{Z}} g^{n}(U)$ with different indices. Since $f \in \mathcal{G}_{3}, f$ has two different periodic orbit $p$ and $q$ in $\Lambda$ with different indices. Since $f$ satisfies condition (P) and $f$ is a Kupka-Samle diffeomorphism, this is a contradiction.

Denote by $\mathcal{F}(M)$ the set of $f \in \operatorname{Diff}(M)$ such that there is a $C^{1}$ neighborhood $\mathcal{U}(f)$ of $f$ such that for any $g \in \mathcal{U}(f)$ and every $p \in P(g)$ is hyperbolic. In [7], Hayashi proved that $f \in \mathcal{F}(M)$ if and only if $f$ satisfies both Axiom $\mathrm{A}$ and no-cycle condition. To prove Theorem 1.5, it is enough to show $f \in \mathcal{F}(M)$. Then we get Theorem 2.1.

End of proof of Theorem 1.5. Let $f \in \mathcal{G}=\mathcal{G}_{2} \cap \mathcal{G}_{3}$ and let $\mathcal{U}(f)$ be a $C^{1}$ neighborhood $\mathcal{U}(f)$ of $f$. The proof is by contradiction. Suppose that $f \notin$ $\mathcal{F}(M)$. Then there exist $g \in \mathcal{U}(f)$ and $\delta>0$ such that non-hyperbolic periodic point $p_{g} \in P(g)$ and $p_{g}$ has a $\delta / 2$ weak eigenvalue. By Remark 2.3, $f$ has a $\delta$-weak eigenvalue which is a contradiction, by Proposition 2.5. The theorem is proved.

Acknowledgments. The first author is supported by Basic Science Research Program through the National Research Foundation of Korea(NRF) funded by the Ministry of Education, Science and Technology, Korea (No. 2011-0007649). The second author is supported by the National Research Foundation (NRF) of Korea funded by the Korean Government (No. 2011-0015193).

\section{References}

1] A. Arbieto, Periodic orbits and expansiveness, Math. Z. 269 (2011), no. 3-4, 801-807.

[2] C. Bonatti and L. J. Díaz, Persistent nonhyperbolic transitive diffeomorphisms, Ann. of Math. (2) 143 (1996), no. 2, 357-396.

[3] C. Bonatti, L. J. Díaz, and E. R. Pujals, A $C^{1}$-generic dichotomy for diffeomorphisms: weak forms of hyperbolicity or infinitlely many sinks or sources, Ann. of Math. 158 (2003), no. 2, 355-418.

[4] M. Carvalho, Sinai-Ruelle-Bowen measures for $N$-dimensional derived freom Anosov diffeomorphisms, Ergodic Theory Dynam. Systems 13 (1993), no. 1, 21-44.

[5] L. J. Díaz, Robust nonhyperbolic dynamics and heterodimensional cycles, Ergodic Theory Dynam. Systems 15 (1995), no. 2, 291-315.

[6] L. J. Díaz, E. R. Pujals, and R. Ures, Partial hyperbolicity and robust transitivity, Acta Math. 183 (1999), no. 1, 1-43.

[7] S. Hayashi, Diffeomorphisms in $\mathcal{F}^{1}(M)$ satisfy Axiom A, Ergodic Theory Dynam. Systems 12 (1992), no. 2, 233-253.

[8] R. Mãné, Contributions to the stability conjecture, Topology 17 (1978), no. 4, 383-396.

[9] _ An ergodic closing lemma, Ann. of Math. (2) 116 (1982), no. 3, 503-540.

[10] K. Sakai, N. Sumi, and K. Yamamoto, Diffeomorphisms satisfying the specification property, Proc. Amer. Math. Soc. 138 (2010), no. 1, 315-321. 
[11] M. Shub, Topologically transitive diffeomorphism of $\mathbb{T}^{4}$, in Symposium on Differential equations and Dyanmical Systems (University of Warwick, 1968/69), pp. 39-40, Lecture Notes in Math., 206. Springer-Verlag, Berlin-New York, 1971.

[12] R. F. Williams, The "DA" maps of Smale and structural stability, in Global Analysis(Berkeley, CA, 1968), pp 329-334, Proc. Sympos. Pure Math., 124. Amer. Math. Soc., Providence, RI, 1970.

[13] D. Yang and S. Gan, Expansive homoclinic classes, Nonlinearity 22 (2009), no. 4, 729733.

MANSEOB Lee

Department of Mathematics

MOKWON UNIVERSITY

DAEJEON 302-729, KoREA

E-mail address: lmsds@mokwon.ac.kr

Seunghee Lee

Department of Mathematics

Chungnam National University

DAEJEON 305-764, Korea

E-mail address: shlee@cnu.ac.kr 